\title{
ESTUDO DA INFLUÊNCIA DOS PARÂMETROS DA TÉCNICA DE INVERSÃO DE FASES NA SÍNTESE DE MEMBRANAS DE POLIETERSULFONA (PES)
}

\author{
F. F. GHIGGI, L. D. POLLO, N. S. M. CARDOZO e I. C. TESSARO \\ Universidade Federal do Rio Grande do Sul, Departamento de Engenharia Química \\ E-mail para contato: nandaghiggi@gmail.com
}

\begin{abstract}
RESUMO - Membranas de PES foram sintetizadas pela técnica de inversão de fases, variando-se os seguintes parâmetros: tempo de evaporação (30 e 60 s) e composição do banho de coagulação (água destilada, W, e água destilada com 5\% v/v do solvente dimetilacetamida, WS). As membranas foram caracterizadas quanto à estrutura por microscopia eletrônica de varredura (MEV), permeância hidráulica e massa molar de corte (MMC). Os resultados indicaram que a membrana PES-30W apresentou características de microfiltração, com permeância hidráulica de $110 \mathrm{~L} \cdot \mathrm{m}^{-2} \cdot \mathrm{h}^{-1} \cdot \mathrm{bar}^{-1}$. As membranas PES-60W e PES-30WS apresentam características de ultrafiltração, com permeância de 29 e $21 \mathrm{~L} \cdot \mathrm{m}^{-2} \cdot \mathrm{h}^{-1} \cdot \mathrm{bar}^{-1}$ e MMC de 17 e $21 \mathrm{kDa}$, respectivamente. O aumento do tempo de evaporação e a adição de solvente no banho de coagulação promoveram uma redução significativa do tamanho dos poros e da permeância hidráulica da membrana.
\end{abstract}

\section{INTRODUÇÃO}

A inversão de fases é a técnica mais utilizada na síntese de membranas poliméricas devido à sua versatilidade, pois possibilita uma grande variedade de morfologias. Esta técnica consiste na passagem, de forma controlada, do polímero em fase líquida para a fase sólida, causada por uma desestabilização termodinâmica da solução polimérica, que induz a separação em duas fases líquidas, uma rica e outra pobre em polímero. A fase rica em polímero dará origem à estrutura da membrana, enquanto a fase pobre aos poros. A desestabilização pode ocorrer de diversas formas: evaporação do solvente, adição de um não-solvente para o polímero ou mudança de temperatura da solução (Mulder, 1996; Habert et al., 2006). O método mais utilizado para fabricação de membranas porosas assimétricas é o de precipitação do polímero, a partir de um filme obtido pelo espalhamento da solução polimérica sobre uma placa de vidro, por imersão em um banho de não-solvente. A etapa de imersão pode ser antecedida de exposição do filme líquido ao ar, para evaporação parcial do solvente. Inicialmente, ocorre evaporação do solvente simultaneamente à absorção da umidade do ar pela solução polimérica. Quando a solução é mergulhada em banho de não-solvente, o solvente migra para o banho e o não-solvente para a solução polimérica (Barth et al., 2000). Um esquema pode ser visualizado na Figura 1. Quanto mais volátil for o solvente, maior o fluxo de evaporação na primeira etapa do processo, e quanto maior a afinidade do solvente com a água, maior o fluxo de migração deste para o banho. 


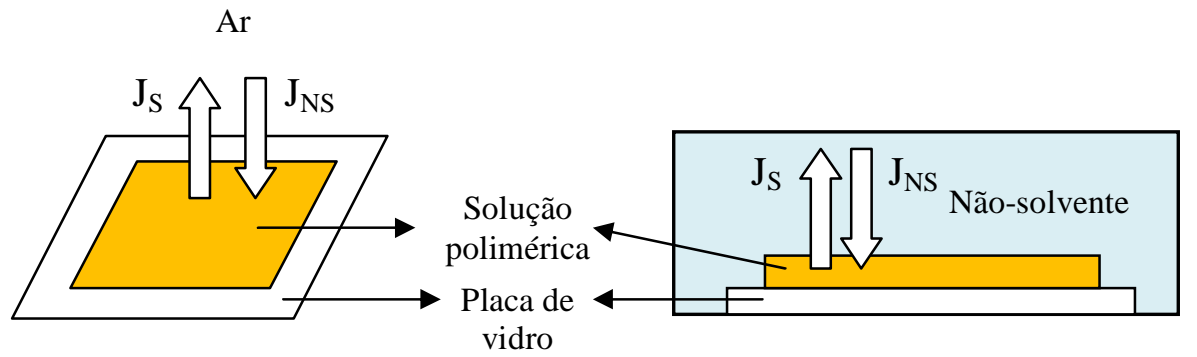

Figura 1 - Esquema da inversão de fases por evaporação do solvente seguida de precipitação em banho de coagulação. $\mathrm{J}_{\mathrm{S}}$, fluxo de solvente; $\mathrm{J}_{\mathrm{NS}}$, fluxo de não-solvente.

De acordo com a estrutura que se deseja obter, podem-se variar os parâmetros do processo, como a concentração do polímero na solução, o tipo de solvente, o tempo de evaporação, a composição do banho de coagulação, além da utilização de aditivos, entre outros (Barth et al., 2000). Muitos autores têm estudado a influência desses parâmetros nas características das membranas. Arthanareeswaran e Starov (2011) sintetizaram membranas de polietersulfona (PES) com diferentes solventes, como dimetilformamida (DMF), dimetilsulfóxido (DMSO) e N-metilpirrolidona (NMP), e avaliaram o efeito do solvente e da concentração de polímero na sua estrutura, características e desempenho. Barth et al. (2000) estudaram a influência da concentração de polímero, do uso de acetona como aditivo, do material do suporte e da espessura de espalhamento do filme na estrutura e propriedade de membranas de polissulfona (PSf) e PES, utilizando DMF como solvente. MosquedaJimenez et al. (2004) investigaram o efeito da concentração de polímero, do tempo de evaporação, da adição de macromoléculas modificadoras de superfície e do uso do aditivo polivinilpirrolidona (PVP) na síntese de membranas de PES, utilizando NMP como solvente.

O objetivo deste trabalho é estudar os efeitos do tempo de evaporação e da composição do banho de coagulação na morfologia, permeabilidade e massa molar de corte (MMC) de membranas de PES produzidas a partir de soluções do polímero em N,N-dimetilacetamida (DMAc).

\section{MATERIAIS E MÉTODOS}

\subsection{Materiais}

A polietersulfona (Ultrason E $6020 \mathrm{P}, \mathrm{M}_{\mathrm{w}}=75 \mathrm{kDa}$ ), gentilmente cedida pela BASF, foi previamente seca em estufa a $60{ }^{\circ} \mathrm{C}$. Na síntese das membranas foi utilizado o solvente $\mathrm{N}, \mathrm{N}$ dimetilacetamida 99\% da Sigma-Aldrich. Para a determinação da MMC das membranas produzidas utilizou-se polietilenoglicol (PEG) de diferentes massas molares (10, 15 e $35 \mathrm{kDa})$ da Merck. 


\subsection{Síntese das Membranas}

As soluções poliméricas foram preparadas dissolvendo-se PES $(15 \% \mathrm{~m} / \mathrm{m})$ em DMAc com agitação magnética, à temperatura ambiente. Antes do espalhamento, as soluções foram colocadas em banho de ultrassom por 30 min para remoção das bolhas de ar. As soluções foram espalhadas sobre placas de vidro utilizando uma faca de espalhamento com espessura de $200 \mu \mathrm{m}$. Deixou-se evaporar o solvente em capela por um tempo determinado e, então, as placas foram imersas em banho de coagulação à temperatura ambiente. A Tabela 1 apresenta o tempo de evaporação e a composição do banho de coagulação de cada membrana. As membranas foram então lavadas e armazenadas em água destilada para remoção do solvente residual.

Tabela 1 - Condições de síntese das membranas

\begin{tabular}{ccc}
\hline Membrana & $\begin{array}{c}\text { Tempo de } \\
\text { evaporação }(\mathbf{s})\end{array}$ & $\begin{array}{c}\text { Composição do banho de } \\
\text { coagulação }(\% \text { v/v })\end{array}$ \\
\hline PES-30W & 30 & Água destilada \\
PES-60W & 60 & Água destilada \\
PES-30WS & 30 & Água destilada/DMAc $(95 / 5)$ \\
\hline
\end{tabular}

\subsection{Caracterização das Membranas}

Morfologia: A morfologia das membranas foi analisada em microscópio eletrônico de varredura (MEV) Hitachi TM 3000 (15 kV, sem metalização) e JEOL JSM 6060 (10 e $15 \mathrm{kV}$, recobrimento com ouro). Para o preparo das amostras, as membranas foram secas pela troca de solventes, método que consiste na imersão da membrana em não-solventes com tensões superficiais decrescentes, a fim de evitar o colapso dos poros. Assim, as membranas úmidas foram imersas em etanol por $3 \mathrm{~h}$ e, posteriormente, em hexano por mais $3 \mathrm{~h}$. Após, as membranas foram deixadas em capela durante a noite para evaporação do hexano. Para a análise da seção transversal, as amostras foram fraturadas em $\mathrm{N}_{2}$ líquido.

Permeância hidráulica: As membranas foram analisadas quanto à permeância hidráulica em uma célula de filtração tangencial. Neste teste, mediu-se o fluxo permeado em diferentes pressões. A permeância foi calculada pela Equação 1 , onde $J$ é o fluxo permeado, $L_{p}$ a permeância hidráulica, e $\Delta P$ a pressão transmembrana.

$$
J=L_{p} \Delta P
$$

Massa molar de corte: É a massa molar que apresenta $90 \%$ de retenção pela membrana. Os testes foram realizados também em célula de filtração tangencial, utilizando soluções de PEG de diferentes massas molares, com concentração inicial de aproximadamente 1000 ppm. A pressão transmembrana utilizada foi de 2,2 bar. Após 15 min de operação em reciclo total, foram retiradas amostras da corrente de concentrado e permeado. A concentração das amostras foi medida em um analisador de carbono orgânico total (Shimadzu TOC-L CPH/CPN). A retenção foi calculada segundo a Equação 2, 


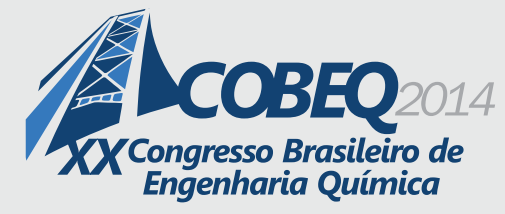

19 a 22 de outubro de 2014

Florianópolis/SC

onde $R$ é a retenção observada, $C_{P}$ a concentração da corrente de permeado, e $C_{C}$ a concentração da corrente de concentrado.

$$
R=1-C_{P} / C_{C}
$$

\section{RESULTADOS E DISCUSSÃO}

\subsection{Morfologia}

Na Figura 2 são apresentadas as fotomicrografias da membrana PES-30W. Com um aumento de 5000x é possível observar os poros na superfície da membrana. Observa-se que o tamanho de poros é da ordem de alguns micrômetros, sendo característico de membranas de microfiltração. A partir do corte transversal, percebe-se que a membrana possui estrutura assimétrica: uma fina camada densificada no topo, que age como barreira seletiva, uma espessa camada intermediária com estrutura em forma de dedos ("finger-like") e uma camada esponjosa na parte inferior.

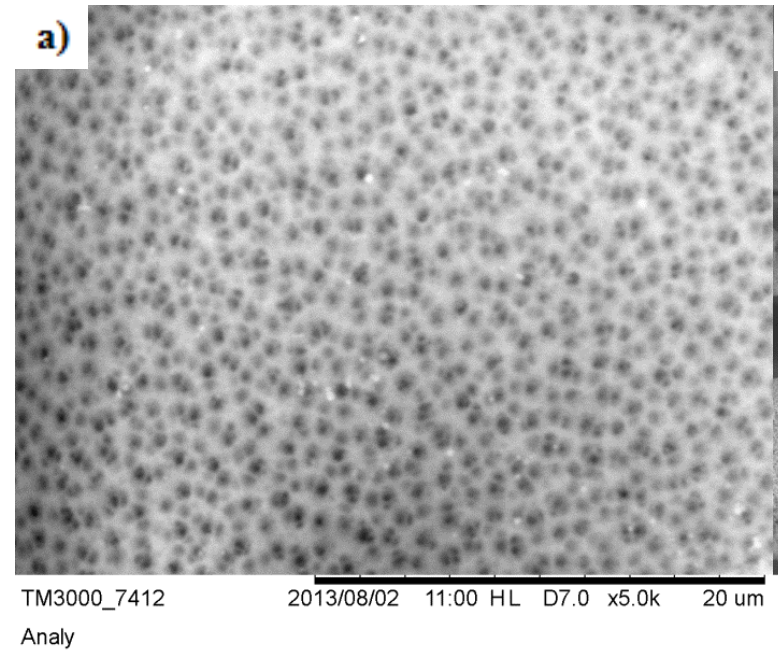

b)

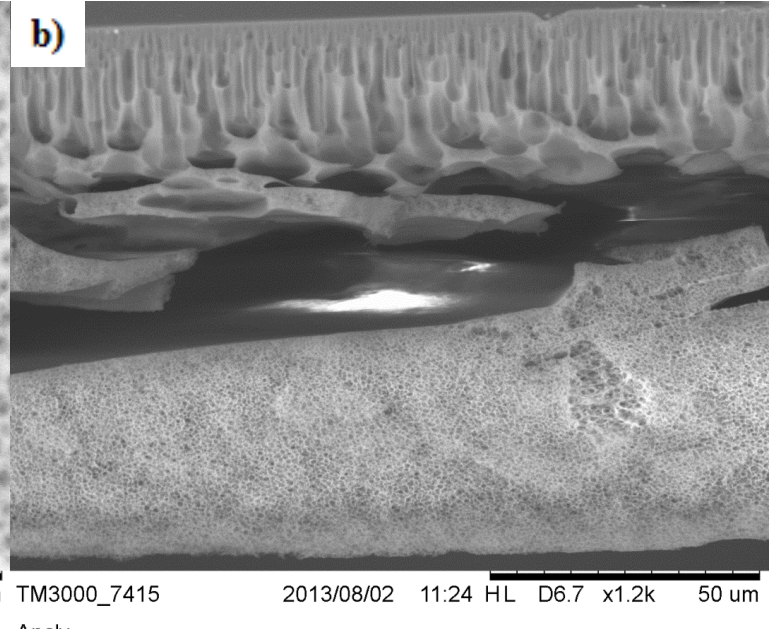

Analy

Figura 2 - Fotomicrografias da (a) superfície (x5000) e da (b) seção transversal (x1200) da membrana PES-30W (MEV Hitachi TM 3000).

Na Figura 3 são apresentadas as fotomicrografias das membranas PES-60W e PES-30WS. Não foi possível observar os poros das membranas, mesmo com um aumento de 40000x, indicando que essas membranas apresentam tamanhos de poros menores que a faixa de tamanhos para a microfiltração. Este resultado indica que tanto o aumento do tempo de evaporação de 30 para $60 \mathrm{~s}$ quanto a adição de $5 \%$ v/v de solvente no banho de coagulação causaram uma redução significativa do tamanho dos poros. Com o aumento do tempo de evaporação, a concentração do polímero na interface filme/ar aumenta, formando uma fina camada densa. Essa densificação da superfície aumenta a resistência à transferência de massa entre o seio da solução polimérica e o banho de 


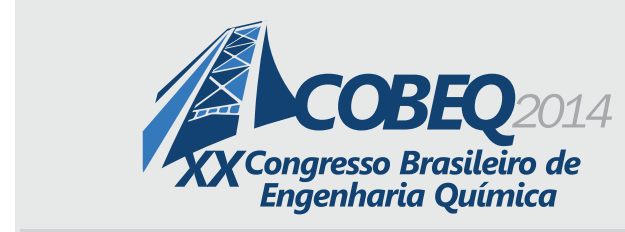

19 a 22 de outubro de 2014

Florianópolis/SC

coagulação, ou seja, os fluxos de saída de solvente e entrada de não-solvente diminuem, causando um atraso no mecanismo de separação de fases e, consequentemente, uma redução do tamanho dos poros (Holda et al., 2013). Já a adição de solvente no banho de coagulação leva a dois efeitos opostos. Devido ao menor gradiente de concentração de solvente, o fluxo deste diminui, causando atraso no mecanismo de separação líquido-líquido e, portanto, redução do tamanho dos poros. No entanto a presença de solvente no banho de coagulação causa diminuição da concentração de polímero na interface filme/banho, produzindo poros maiores (Mulder, 1996). Neste trabalho, o primeiro efeito foi mais significativo, uma vez que se observou a redução do tamanho dos poros.

Quanto à morfologia, ambas as membranas apresentaram estruturas assimétricas semelhantes, caracterizadas principalmente por uma fina camada seletiva no topo e macrovazios em forma de dedos que se prolongam através da espessura da membrana.

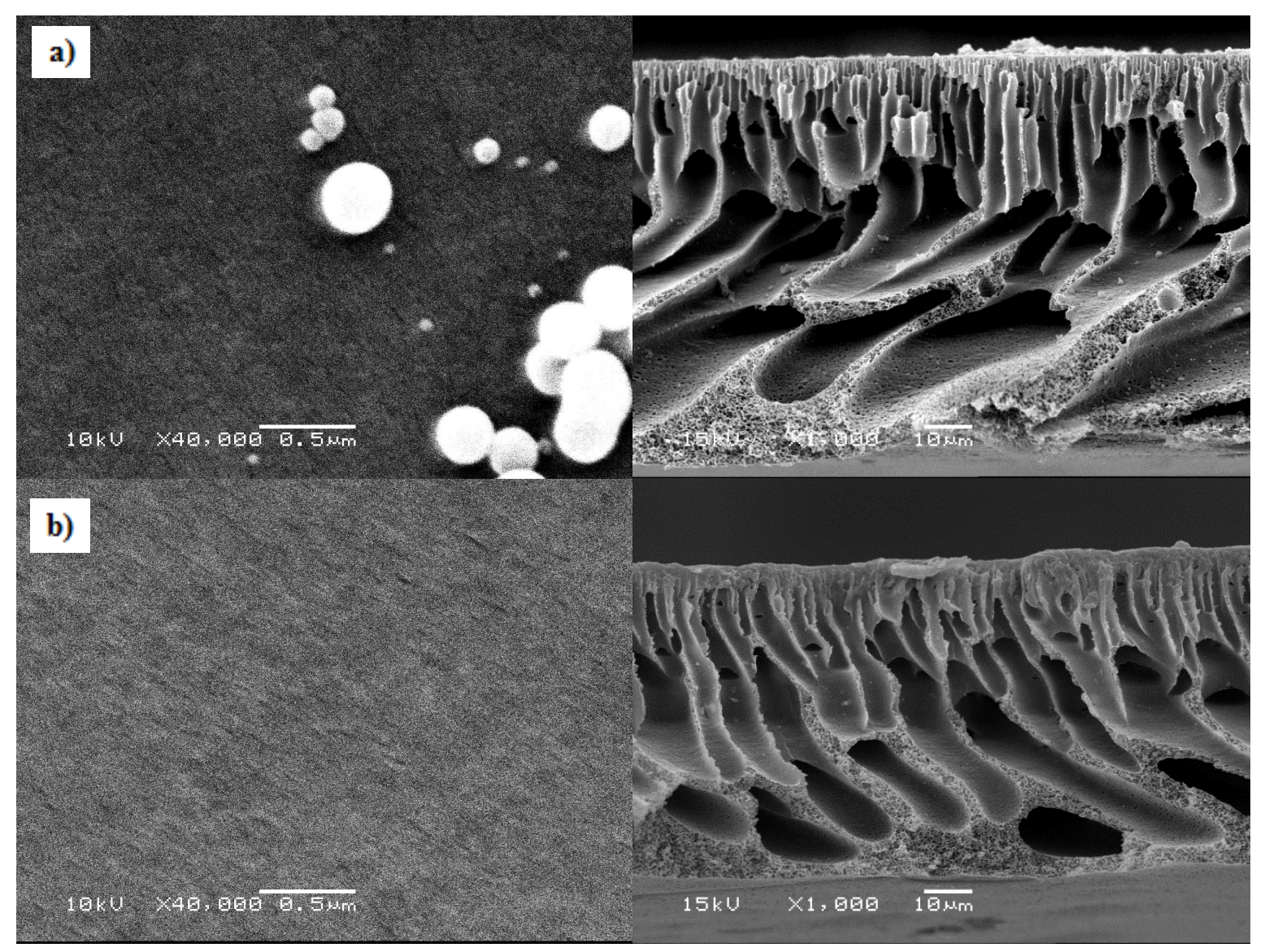

Figura 3 - Fotomicrografias de superfície (x40000) à esquerda e seção transversal (x1000) à direita das membranas (a) PES-60W e (b) PES-30WS (MEV JEOL JSM 6060). 


\subsection{Permeância Hidráulica}

Os resultados do teste de permeância hidráulica são apresentados na Figura 4. A partir dos dados obtidos foram traçadas linhas de tendência, cujos coeficientes angulares correspondem à permeância hidráulica das membranas. Pressões na faixa de 1 a 3 bar foram utilizadas para a membrana PES-30W, que apresentou permeância de $110 \mathrm{~L} \cdot \mathrm{m}^{-2} \cdot \mathrm{h}^{-1} \cdot \mathrm{bar}^{-1}\left(\mathrm{R}^{2}=0,978\right)$. As membranas PES-60W e PES-30WS foram testadas em uma faixa de pressão maior, de 3 a 6 bar, e apresentaram permeância de 29 e $21 \mathrm{~L} \cdot \mathrm{m}^{-2} \cdot \mathrm{h}^{-1} \cdot$ bar $^{-1}\left(\mathrm{R}^{2}=0,990\right.$ e 0,988$)$, respectivamente. A comparação dos valores de permeância hidráulica das membranas PES-60W e PES-30WS com o da membrana PES-30W indica que a adição de solvente no banho de coagulação teve maior influência na redução da permeância comparado ao tempo de evaporação.

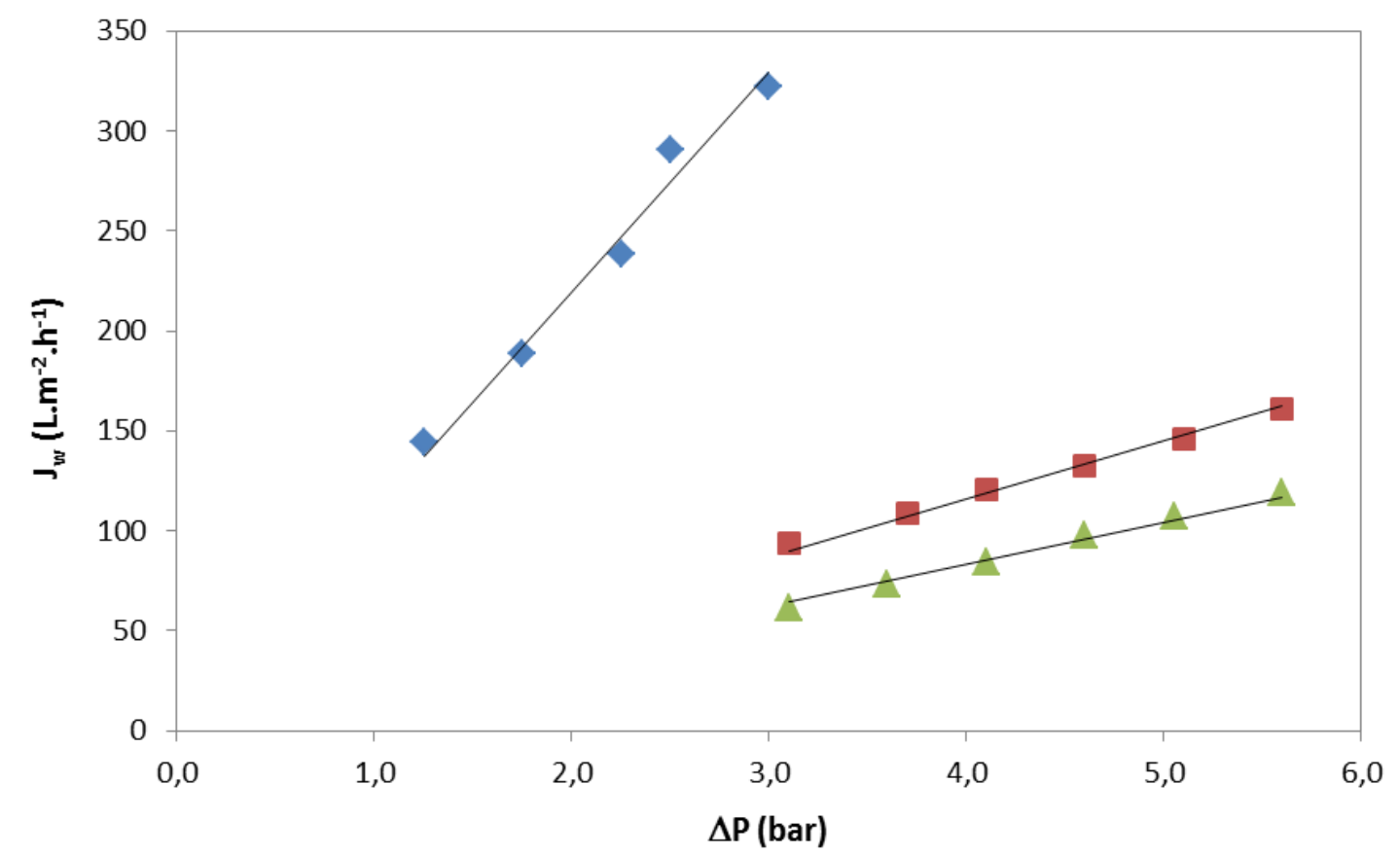



\subsection{Massa Molar de Corte}

Os resultados de retenção de PEG das membranas PES-60W e PES-30WS são mostrados na Figura 5. As curvas foram ajustadas pelo modelo log-normal (Van der Bruggen et al., 2000) para determinação da MMC dessas membranas. As membranas PES-60W e PES-30WS apresentaram valores aproximados de $\mathrm{MMC}$ de 17 e $21 \mathrm{kDa}\left(\mathrm{R}^{2}=0,971\right.$ e 0,996), respectivamente, os quais correspondem a valores típicos de membranas de ultrafiltração. 


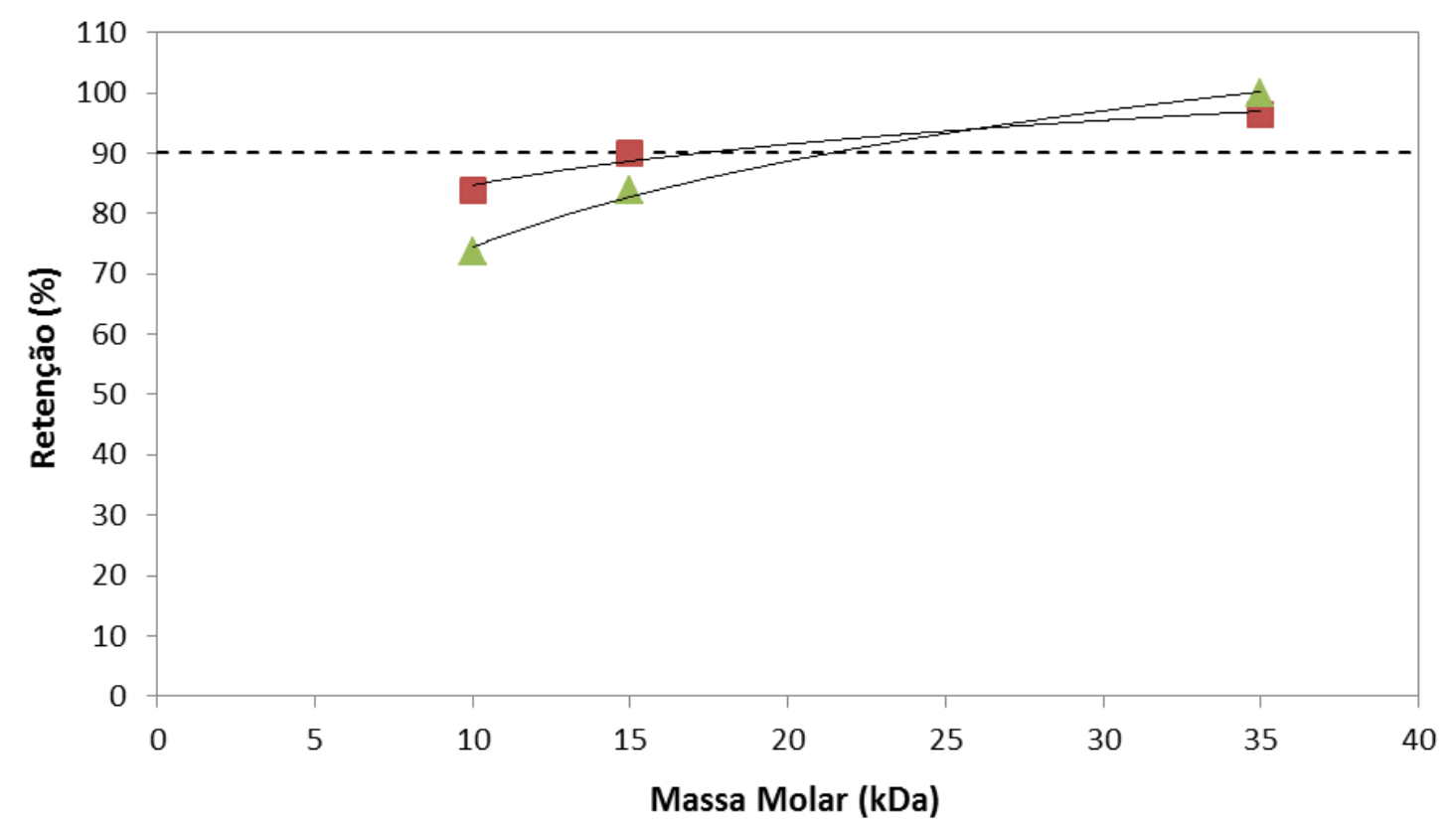

Figura 5 - Curvas de retenção das membranas PES-60W (ロ) e PES-30WS (A).

\section{CONCLUSÕES}

Membranas de PES foram sintetizadas pela técnica de inversão de fases, variando-se o tempo de evaporação e a composição do banho de coagulação, e caracterizadas quanto à morfologia, permeância hidráulica e massa molar de corte.

A membrana para a qual se utilizou somente água na composição do banho de coagulação e uma etapa de evaporação mais curta (PES-30W) apresentou características mais próximas às de uma membrana de microfiltração.

Tanto o aumento do tempo de evaporação quanto a adição de solvente no banho de coagulação levaram a uma redução significativa do tamanho dos poros, de forma que essas membranas apresentaram características próximas às de uma membrana de ultrafiltração em termos de permeância hidráulica e massa molar de corte. Apesar de que não foram medidos a distribuição e o tamanho dos poros nas três membranas em estudo, estes resultados indicam também que o efeito da adição de solvente no banho de coagulação na redução do tamanho de poro é maior do que aquele exercido pelo aumento do tempo de evaporação. 


\section{REFERÊNCIAS}

ARTHANAREESWARAN, G.; STAROV, V. M. Effect of solvents on performance of polyethersulfone ultrafiltration membranes: Investigation of metal ion separations, Desalination, v. 267, p. 57-63, 2011.

BARTH, C.; GONÇALVES, M. C.; PIRES, A. T. N.; ROEDER, J.; WOLF, B. A. Asymmetric polysulfone and polyethersulfone membranes: effects of thermodynamic conditions during formation on their performance, Journal of Membrane Science, v. 169, p. 287-299, 2000.

HABERT, A. C.; BORGES, C. P.; NOBREGA, R. Processos de Separação por Membranas. Rio de Janeiro: E-papers, 2006.

HOLDA, A. K.; AERNOUTS, B.; SAEYS, W.; VANKELECOM, I. F. J. Study of polymer concentration and evaporation time as phase inversion parameters for polysulfone-based SRNF membranes. Journal of Membrane Science, v. 442, p. 196-205, 2013.

MOSQUEDA-JIMENEZ, D. B.; NARBAITZ, R. M.; MATSUURA, T.; CHOWDHURY, G.; G. PLEIZIER, G.; SANTERRE, J. P. Influence of processing conditions on the properties of ultrafiltration membranes. Journal of Membrane Science, v. 231, p. 209-224, 2004.

MULDER, M. Basic Principles of Membrane Technology. Dordrecht: Kluwer Academic Publishers, 1996.

VAN DER BRUGGEN, B.; SCHAEP, J.; WILMS, D.; VANDECASTEELE, C. A comparison of models to describe the maximal retention of organic molecules in nanofiltration, Separation Science and Technology, v. 35, p. 169-182, 2000. 